\title{
Strong Differential Sandwich Results of $\lambda$-Pseudo-Starlike Functions with Respect to Symmetrical Points
}

\author{
H. M. SRivastava, Abbas Kareem Wanas
}

\begin{abstract}
In the present investigation, by considering suitable classes of admissible functions, we establish strong differential subordination and superordination properties for $\lambda$-pseudo-starlike functions with respect to symmetrical points in the open unit disk $U$. These results are applied to obtain strong differential sandwich results.
\end{abstract}

\section{Introduction AND PRELIMINARIES}

Let $\mathcal{H}(U)$ be the class of analytic functions in the open unit disk $U=$ $\{z \in \mathbb{C}:|z|<1\}$. For a positive integer $n$ and $a \in \mathbb{C}$, let $\mathcal{H}[a, n]$ be the subclass of $\mathcal{H}(U)$ consisting of functions of the form:

$$
f(z)=a+a_{n} z^{n}+a_{n+1} z^{n+1}+\cdots,
$$

with $\mathcal{H}=\mathcal{H}[1,1]$.

Let $\mathcal{A}$ stand for the class of all analytic functions in $U$ and having the form:

$$
f(z)=z+\sum_{n=2}^{\infty} a_{n} z^{n}, \quad(z \in U),
$$

A function $f \in \mathcal{A}$ is called starlike with respect to symmetrical points, if (see [10])

$$
\operatorname{Re}\left\{\frac{z f^{\prime}(z)}{f(z)-f(-z)}\right\}>0, \quad z \in U .
$$

The set of all such functions is denote by $S_{s}^{*}$.

Recently, Babalola [2] defined the class $\mathcal{L}_{\lambda}$ of $\lambda$-pseudo-starlike functions as follows:

2010 Mathematics Subject Classification. Primary: 30C45; Secondary: 30A20.

Key words and phrases. Strong differential subordination, Strong differential superordination, $\lambda$-pseudo-starlike functions, symmetrical points, Admissible functions.

Full paper. Received 11 June 2019, revised 30 September 2019, accepted 13 October 2019, available online 18 October 2019. 
Let $f \in \mathcal{A}$ and $\lambda \geq 1$. Then $f \in \mathcal{L}_{\lambda}$ of $\lambda$-pseudo-starlike functions in $U$ if and only if

$$
\operatorname{Re}\left\{\frac{z\left(f^{\prime}(z)\right)^{\lambda}}{f(z)}\right\}>0, \quad z \in U .
$$

A function $f \in \mathcal{A}$ is called $\lambda$-pseudo-starlike with respect to symmetrical points, if

$$
\operatorname{Re}\left\{\frac{z\left(f^{\prime}(z)\right)^{\lambda}}{f(z)-f(-z)}\right\}>0, \quad z \in U .
$$

We denote by $\mathcal{L}_{\lambda, s}^{*}$ the family of all $\lambda$-pseudo-starlike functions with respect to symmetrical points.

Let $f$ and $g$ be members of $\mathcal{H}(U)$. The function $f$ is said to be subordinate to $g$, or (equivalently) $g$ is said to be superordinate to $f$, if there exists a Schwarz function $w$ which is analytic in $U$ with $w(0)=0$ and $|w(z)|<$ $1(z \in U)$ such that $f(z)=g(w(z))$. In such a case, we write $f \prec g$ or $f(z) \prec g(z)(z \in U)$. Furthermore, if the function $g$ is univalent in $U$, then we have the following equivalent (see [5])

$$
f(z) \prec g(z) \Longleftrightarrow f(0)=g(0) \text { and } f(U) \subset g(U) .
$$

Let $G(z, \zeta)$ be analytic in $U \times \bar{U}$ and let $f(z)$ be analytic and univalent in $U$. Then the function $G(z, \zeta)$ is said to be strongly subordinate to $f(z)$ or $f(z)$ is said to be strongly superordinate to $G(z, \zeta)$, written as $G(z, \zeta) \prec \prec f(z)$, if for $\zeta \in \bar{U}=\{z \in \mathbb{C}:|z| \leq 1\}, G(z, \zeta)$ as a function of $z$ is subordinate to $f(z)$. We note that

$$
G(z, \zeta) \prec \prec f(z) \Longleftrightarrow G(0, \zeta)=f(0) \text { and } G(U \times \bar{U}) \subset f(U) .
$$

Definition 1.1 ([6]). Let $\phi: \mathbb{C}^{3} \times U \times \bar{U} \longrightarrow \mathbb{C}$ and let $h$ be a univalent function in $U$. If $p$ is analytic in $U$ and satisfies the following (second-order) strong differential subordination:

$$
\phi\left(p(z), z p^{\prime}(z), z^{2} p^{\prime \prime}(z) ; z, \zeta\right) \prec \prec h(z),
$$

then $p$ is called a solution of the strong differential subordination (1). The univalent function $q$ is called a dominant of the solutions of the strong differential subordination or more simply a dominant if $p(z) \prec q(z)$ for all $p$ satisfying (1). A dominant $\check{q}$ that satisfies $\check{q}(z) \prec q(z)$ for all dominants $q$ of (1) is said to be the best dominant.

Definition $1.2([7])$. Let $\phi: \mathbb{C}^{3} \times U \times \bar{U} \longrightarrow \mathbb{C}$ and let $h$ be analytic function in $U$. If $p$ and $\phi\left(p(z), z p^{\prime}(z), z^{2} p^{\prime \prime}(z) ; z, \zeta\right)$ are univalent in $U$ for $\zeta \in \bar{U}$ and satisfy the following (second-order) strong differential superordination:

$$
h(z) \prec \prec \phi\left(p(z), z p^{\prime}(z), z^{2} p^{\prime \prime}(z) ; z, \zeta\right),
$$

then $p$ is called a solution of the strong differential superordination (2). An analytic function $q$ is called a subordinant of the solutions of the strong differential superordination or more simply a subordinant if $q(z) \prec p(z)$ for 
all $p$ satisfying (2). A univalent subordinant $\check{q}$ that satisfies $q(z) \prec \breve{q}(z)$ for all subordinants $q$ of (2) is said to be the best subordinant.

Definition 1.3 ([6]). Denote by $Q$ the set consisting of all functions $q$ that are analytic and injective on $\bar{U} \backslash E(q)$, where

$$
E(q)=\left\{\xi \in \partial U: \lim _{z \rightarrow \xi} q(z)=\infty\right\},
$$

and are such that $q^{\prime}(\xi) \neq 0$ for $\xi \in \partial U \backslash E(q)$.

Furthermore, let the subclass of $Q$ for which $q(0)=a$ be denoted by $Q(a)$, $Q(0) \equiv Q_{0}$ and $Q(1) \equiv Q_{1}$.

Definition $1.4([9])$. Let $\Omega$ be a set in $\mathbb{C}, q \in Q$ and $n \in \mathbb{N}$. The class of admissible functions $\Psi_{n}[\Omega, q]$ consists of those functions $\psi: \mathbb{C}^{3} \times U \times$ $\bar{U} \longrightarrow \mathbb{C}$ that satisfy the following admissibility condition: $\psi(r, s, t ; z, \zeta) \notin$ $\Omega$, whenever

$$
r=q(\xi), \quad s=k \xi q^{\prime}(\xi) \quad \text { and } \quad \operatorname{Re}\left\{\frac{t}{s}+1\right\} \geq k \operatorname{Re}\left\{\frac{\xi q^{\prime \prime}(\xi)}{q^{\prime}(\xi)}+1\right\},
$$

$z \in U, \xi \in \partial U \backslash E(q), \zeta \in \bar{U}$ and $k \geq n$.

We simply write $\Psi_{1}[\Omega, q]=\Psi[\Omega, q]$.

Definition $1.5([8])$. Let $\Omega$ be a set in $\mathbb{C}$ and $q \in \mathcal{H}[a, n]$ with $q^{\prime}(z) \neq$ 0 . The class of admissible functions $\Psi_{n}^{\prime}[\Omega, q]$ consists of those functions $\psi: \mathbb{C}^{3} \times U \times \bar{U} \longrightarrow \mathbb{C}$ that satisfy the following admissibility condition: $\psi(r, s, t ; \xi, \zeta) \in \Omega$, whenever

$$
r=q(z), \quad s=\frac{z q^{\prime}(z)}{m} \quad \text { and } \quad \operatorname{Re}\left\{\frac{t}{s}+1\right\} \leq \frac{1}{m} \operatorname{Re}\left\{\frac{z q^{\prime \prime}(z)}{q^{\prime}(z)}+1\right\},
$$

$z \in U, \xi \in \partial U, \zeta \in \bar{U}$ and $m \geq n \geq 1$.

In particular, we write $\Psi_{1}^{\prime}[\Omega, q]=\Psi^{\prime}[\Omega, q]$.

In our investigations, we will need the following lemmas:

Lemma $1.1([9])$. Let $\psi \in \Psi_{n}[\Omega, q]$ with $q(0)=a$. If $p \in \mathcal{H}[a, n]$ satisfies

$$
\psi\left(p(z), z p^{\prime}(z), z^{2} p^{\prime \prime}(z) ; z, \zeta\right) \in \Omega,
$$

then $p(z) \prec q(z)$.

Lemma $1.2([8])$. Let $\psi \in \Psi_{n}^{\prime}[\Omega, q]$ with $q(0)=a$. If $p \in Q(a)$ and $\psi\left(p(z), z p^{\prime}(z), z^{2} p^{\prime \prime}(z) ; z, \zeta\right)$ is univalent in $U$ for $\zeta \in \bar{U}$, then

$$
\Omega \subset\left\{\psi\left(p(z), z p^{\prime}(z), z^{2} p^{\prime \prime}(z) ; z, \zeta\right): z \in U, \zeta \in \bar{U}\right\}
$$

implies $q(z) \prec p(z)$.

In recent years, several authors obtained many interesting results in strong differential subordination and superordination $[1,3,4,11,12,13]$. In this work, by making use of the strong differential subordination results and 
strong differential superordination results of Oros and Oros [8, 9], we introduce and study certain suitable classes of admissible functions and derive some strong differential subordination and superordination properties of $\lambda$ pseudo-starlike functions with respect to symmetrical points.

\section{Strong Subordination Results}

Definition 2.1. Let $\Omega$ be a set in $\mathbb{C}$ and $q \in Q_{1} \cap \mathcal{H}$. The class of admissible functions $\Phi_{\mathcal{L}}[\Omega, q]$ consists of those functions $\phi: \mathbb{C}^{3} \times U \times \bar{U} \longrightarrow \mathbb{C}$ that satisfy the admissibility condition: $\phi(u, v, w ; z, \zeta) \notin \Omega$, whenever $u=q(\xi), v=\frac{k \xi q^{\prime}(\xi)}{q(\xi)}, q(\xi) \neq 0$ and $\operatorname{Re}\left\{\frac{w+v^{2}}{v}\right\} \geq k \operatorname{Re}\left\{\frac{\xi q^{\prime \prime}(\xi)}{q^{\prime}(\xi)}+1\right\}$,

where $z \in U, \zeta \in \bar{U}, \xi \in \partial U \backslash E(q)$ and $k \geq 1$.

Theorem 2.1. Let $\phi \in \Phi_{\mathcal{L}}[\Omega, q]$. If $f \in \mathcal{A}$ satisfies

$$
\begin{aligned}
& \left\{\phi \left(\frac{2 z\left(f^{\prime}(z)\right)^{\lambda}}{f(z)-f(-z)}, 1+\frac{\lambda z f^{\prime \prime}(z)}{f^{\prime}(z)}-\frac{z(f(z)-f(-z))^{\prime}}{f(z)-f(-z)}, \frac{\lambda z^{2} f^{\prime \prime \prime}(z)}{f^{\prime}(z)}\right.\right. \\
+ & \frac{\lambda z f^{\prime \prime}(z)}{f^{\prime}(z)}\left(1-\frac{z f^{\prime \prime}(z)}{f^{\prime}(z)}\right)-\frac{z^{2}(f(z)-f(-z))^{\prime \prime}}{f(z)-f(-z)}+\frac{z(f(z)-f(-z))^{\prime}}{f(z)-f(-z)} \times \\
\times & \left.\left.\left(\frac{z(f(z)-f(-z))^{\prime}}{f(z)-f(-z)}-1\right) ; z, \zeta\right): z \in U, \zeta \in \bar{U}\right\} \subset \Omega,
\end{aligned}
$$

then

$$
\frac{2 z\left(f^{\prime}(z)\right)^{\lambda}}{f(z)-f(-z)} \prec q(z) .
$$

Proof. We define the function $p$ by

$$
p(z)=\frac{2 z\left(f^{\prime}(z)\right)^{\lambda}}{f(z)-f(-z)} .
$$

It is clear that $p$ is analytic in $U$.

Simple calculations from (4), we obtain

$$
\frac{z p^{\prime}(z)}{p(z)}=1+\frac{\lambda z f^{\prime \prime}(z)}{f^{\prime}(z)}-\frac{z(f(z)-f(-z))^{\prime}}{f(z)-f(-z)} .
$$

Further computations show that

$$
\begin{aligned}
& \frac{z^{2} p^{\prime \prime}(z)}{p(z)}+\frac{z p^{\prime}(z)}{p(z)}-\left(\frac{z p^{\prime}(z)}{p(z)}\right)^{2}=z\left[1+\frac{\lambda z f^{\prime \prime}(z)}{f^{\prime}(z)}-\frac{z(f(z)-f(-z))^{\prime}}{f(z)-f(-z)}\right]^{\prime} \\
(6)= & \frac{\lambda z^{2} f^{\prime \prime \prime}(z)}{f^{\prime}(z)}+\frac{\lambda z f^{\prime \prime}(z)}{f^{\prime}(z)}\left(1-\frac{z f^{\prime \prime}(z)}{f^{\prime}(z)}\right)-\frac{z^{2}(f(z)-f(-z))^{\prime \prime}}{f(z)-f(-z)} \\
+ & \frac{z(f(z)-f(-z))^{\prime}}{f(z)-f(-z)}\left(\frac{z(f(z)-f(-z))^{\prime}}{f(z)-f(-z)}-1\right) .
\end{aligned}
$$


Define the transforms from $\mathbb{C}^{3}$ to $\mathbb{C}$ by

$$
u=r, \quad v=\frac{s}{r}, \quad w=\frac{r(t+s)-s^{2}}{r^{2}} .
$$

Let

$$
\psi(r, s, t ; z, \zeta)=\phi(u, v, w ; z, \zeta)=\phi\left(r, \frac{s}{r}, \frac{r(t+s)-s^{2}}{r^{2}} ; z, \zeta\right) .
$$

The proof shall make use of Lemma 1.1. Using equations (4), (5) and (6), it follows from (7) that

$$
\begin{aligned}
& \psi\left(p(z), z p^{\prime}(z), z^{2} p^{\prime \prime}(z) ; z, \zeta\right) \\
= & \phi\left(\frac{2 z\left(f^{\prime}(z)\right)^{\lambda}}{f(z)-f(-z)}, 1+\frac{\lambda z f^{\prime \prime}(z)}{f^{\prime}(z)}-\frac{z(f(z)-f(-z))^{\prime}}{f(z)-f(-z)}, \frac{\lambda z^{2} f^{\prime \prime \prime}(z)}{f^{\prime}(z)}\right. \\
+ & \frac{\lambda z f^{\prime \prime}(z)}{f^{\prime}(z)}\left(1-\frac{z f^{\prime \prime}(z)}{f^{\prime}(z)}\right)-\frac{z^{2}(f(z)-f(-z))^{\prime \prime}}{f(z)-f(-z)}+\frac{z(f(z)-f(-z))^{\prime}}{f(z)-f(-z)} \times \\
\times & \left.\left(\frac{z(f(z)-f(-z))^{\prime}}{f(z)-f(-z)}-1\right) ; z, \zeta\right) .
\end{aligned}
$$

Thus (3) becomes $\psi\left(p(z), z p^{\prime}(z), z^{2} p^{\prime \prime}(z) ; z, \zeta\right) \in \Omega$.

To complete the proof, we next show that the admissibility condition for $\phi \in \Phi_{\mathcal{L}}[\Omega, q]$ is equivalent to the admissibility condition for $\psi$ as given in Definition 1.4 .

Note that

$$
\frac{t}{s}+1=\frac{w+v^{2}}{v}
$$

Hence $\psi \in \Psi[\Omega, q]$. By Lemma 1.1, $p(z) \prec q(z)$ or equivalently

$$
\frac{2 z\left(f^{\prime}(z)\right)^{\lambda}}{f(z)-f(-z)} \prec q(z)
$$

We consider the special situation when $\Omega \neq \mathbb{C}$ is a simply connected domain. In this case $\Omega=h(U)$, for some conformal mapping $h$ of $U$ onto $\Omega$ and the class $\Phi_{\mathcal{L}}[h(U), q]$ is written as $\Phi_{\mathcal{L}}[h, q]$. The following result is an immediate consequence of Theorem 2.1.

Theorem 2.2. Let $\phi \in \Phi_{\mathcal{L}}[h, q]$. If $f \in \mathcal{A}$ satisfies

$$
\begin{aligned}
& \phi\left(\frac{2 z\left(f^{\prime}(z)\right)^{\lambda}}{f(z)-f(-z)}, 1+\frac{\lambda z f^{\prime \prime}(z)}{f^{\prime}(z)}-\frac{z(f(z)-f(-z))^{\prime}}{f(z)-f(-z)}, \frac{\lambda z^{2} f^{\prime \prime \prime}(z)}{f^{\prime}(z)}\right. \\
& +\frac{\lambda z f^{\prime \prime}(z)}{f^{\prime}(z)}\left(1-\frac{z f^{\prime \prime}(z)}{f^{\prime}(z)}\right)-\frac{z^{2}(f(z)-f(-z))^{\prime \prime}}{f(z)-f(-z)}+\frac{z(f(z)-f(-z))^{\prime}}{f(z)-f(-z)} \\
& \left.\times\left(\frac{z(f(z)-f(-z))^{\prime}}{f(z)-f(-z)}-1\right) ; z, \zeta\right) \prec \prec h(z),
\end{aligned}
$$


then

$$
\frac{2 z\left(f^{\prime}(z)\right)^{\lambda}}{f(z)-f(-z)} \prec q(z) .
$$

By taking $\phi(u, v, w ; z, \zeta)=u+\frac{v}{\beta u+\gamma},(\beta, \gamma \in \mathbb{C})$ in Theorem 2.2, we state the following corollary:

Corollary 2.1. Let $\beta, \gamma \in \mathbb{C}$ and let $h$ be convex in $U$ with $h(0)=1$ and $\operatorname{Re}\{\beta h(z)+\gamma\}>0$. If $f \in \mathcal{A}$ satisfies

$$
\frac{2 z\left(f^{\prime}(z)\right)^{\lambda}}{f(z)-f(-z)}+\frac{\left(1+\frac{\lambda z f^{\prime \prime}(z)}{f^{\prime}(z)}\right)(f(z)-f(-z))-z(f(z)-f(-z))^{\prime}}{2 \beta z\left(f^{\prime}(z)\right)^{\lambda}+\gamma(f(z)-f(-z))} \prec \prec h(z),
$$

then

$$
\frac{2 z\left(f^{\prime}(z)\right)^{\lambda}}{f(z)-f(-z)} \prec q(z) .
$$

The next result is an extension of Theorem 2.1 to the case where the behavior of $q$ on $\partial U$ is not known.

Corollary 2.2. Let $\Omega \in \mathbb{C}$ and $q$ be univalent in $U$ with $q(0)=1$. Let $\phi \in \Phi_{\mathcal{L}}\left[h, q_{\rho}\right]$ for some $\rho \in(0,1)$, where $q_{\rho}(z)=q(\rho z)$. If $f \in \mathcal{A}$ satisfies

$$
\begin{aligned}
& \phi\left(\frac{2 z\left(f^{\prime}(z)\right)^{\lambda}}{f(z)-f(-z)}, 1+\frac{\lambda z f^{\prime \prime}(z)}{f^{\prime}(z)}-\frac{z(f(z)-f(-z))^{\prime}}{f(z)-f(-z)}, \frac{\lambda z^{2} f^{\prime \prime \prime}(z)}{f^{\prime}(z)}\right. \\
& +\frac{\lambda z f^{\prime \prime}(z)}{f^{\prime}(z)}\left(1-\frac{z f^{\prime \prime}(z)}{f^{\prime}(z)}\right)-\frac{z^{2}(f(z)-f(-z))^{\prime \prime}}{f(z)-f(-z)}+\frac{z(f(z)-f(-z))^{\prime}}{f(z)-f(-z)} \\
& \left.\left(\frac{z(f(z)-f(-z))^{\prime}}{f(z)-f(-z)}-1\right) ; z, \zeta\right) \in \Omega,
\end{aligned}
$$

then

$$
\frac{2 z\left(f^{\prime}(z)\right)^{\lambda}}{f(z)-f(-z)} \prec q(z) .
$$

Proof. Theorem 2.1 yields $\frac{2 z\left(f^{\prime}(z)\right)^{\lambda}}{f(z)-f(-z)} \prec q_{\rho}(z)$. The result is now deduced from the fact that $q_{\rho}(z) \prec q(z)$.

Theorem 2.3. Let $h$ and $q$ be univalent in $U$ with $q(0)=1$ and set $q_{\rho}(z)=$ $q(\rho z)$ and $h_{\rho}(z)=h(\rho z)$. Let $\phi: \mathbb{C}^{3} \times U \times \bar{U} \longrightarrow \mathbb{C}$ satisfy one of the following conditions:

(1) $\phi \in \Phi_{\mathcal{L}}\left[h, q_{\rho}\right]$ for some $\rho \in(0,1)$,

(2) there exists $\rho_{0} \in(0,1)$ such that $\phi \in \Phi_{\mathcal{L}}\left[h_{\rho}, q_{\rho}\right]$ for all $\rho \in\left(\rho_{0}, 1\right)$.

If $f \in \mathcal{A}$ satisfies (9), then

$$
\frac{2 z\left(f^{\prime}(z)\right)^{\lambda}}{f(z)-f(-z)} \prec q(z) .
$$


Proof. Case (1): By applying Theorem 2.1, we obtain $\frac{2 z\left(f^{\prime}(z)\right)^{\lambda}}{f(z)-f(-z)} \prec q_{\rho}(z)$, since $q_{\rho}(z) \prec q(z)$, we deduce

$$
\frac{2 z\left(f^{\prime}(z)\right)^{\lambda}}{f(z)-f(-z)} \prec q(z) .
$$

Case (2): Let $p(z)=\frac{2 z\left(f^{\prime}(z)\right)^{\lambda}}{f(z)-f(-z)}$ and $p_{\rho}(z)=p(\rho z)$. Then

$\phi\left(p_{\rho}(z), z p_{\rho}^{\prime}(z), z^{2} p_{\rho}^{\prime \prime}(z) ; \rho z, \zeta\right)=\phi\left(p(\rho z), z p^{\prime}(\rho z), z^{2} p^{\prime \prime}(\rho z) ; \rho z, \zeta\right) \in h_{\rho}(U)$.

By using Theorem 2.1 and the comment associated with

$$
\phi\left(p(z), z p^{\prime}(z), z^{2} p^{\prime \prime}(z) ; w(z), \zeta\right) \in \Omega,
$$

where $w$ is any function mapping $U$ into $U$, with $w(z)=\rho z$, we obtain $p_{\rho}(z) \prec q_{\rho}(z)$ for $\rho \in\left(\rho_{0}, 1\right)$. By letting $\rho \rightarrow 1^{-}$, we get $p(z) \prec q(z)$. Therefore

$$
\frac{2 z\left(f^{\prime}(z)\right)^{\lambda}}{f(z)-f(-z)} \prec q(z) .
$$

The next result gives the best dominant of the strong differential subordination (9):

Theorem 2.4. Let $h$ be univalent in $U$ and $\phi: \mathbb{C}^{3} \times U \times \bar{U} \longrightarrow \mathbb{C}$. Suppose that the differential equation

$$
\phi\left(q(z), \frac{z q^{\prime}(z)}{q(z)}, \frac{z^{2} q^{\prime \prime}(z)}{q(z)}+\frac{z q^{\prime}(z)}{q(z)}-\left(\frac{z q^{\prime}(z)}{q(z)}\right)^{2} ; z, \zeta\right)=h(z)
$$

has a solution $q$ with $q(0)=1$ and satisfies one of the following conditions:

(1) $q \in Q_{1}$ and $\phi \in \Phi_{\mathcal{L}}[h, q]$,

(2) $q$ is univalent in $U$ and $\phi \in \Phi_{\mathcal{L}}\left[h, q_{\rho}\right]$ for some $\rho \in(0,1)$,

(3) $q$ is univalent in $U$ and there exists $\rho_{0} \in(0,1)$ such that $\phi \in$ $\Phi_{\mathcal{L}}\left[h_{\rho}, q_{\rho}\right]$ for all $\rho \in\left(\rho_{0}, 1\right)$.

If $f \in \mathcal{A}$ satisfies (9), then

$$
\frac{2 z\left(f^{\prime}(z)\right)^{\lambda}}{f(z)-f(-z)} \prec q(z) .
$$

and $q$ is the best dominant.

Proof. By applying Theorem 2.2 and Theorem 2.3, we deduce that $q$ is a dominant of (9). Since $q$ satisfies (10), it is also a solution of (9) and therefore $q$ will be dominated by all dominants.

Hence $q$ is the best dominant of (9).

In the particular case $q(z)=1+M z, M>0$ and in view of Definition 2.1 , the class of admissible functions $\Phi_{\mathcal{L}}[\Omega, q]$ denoted by $\Phi_{\mathcal{L}}[\Omega, M]$ can be expressed in the following form: 
Definition 2.2. Let $\Omega$ be a set in $\mathbb{C}$ and $M>0$. The class of admissible function $\Phi_{\mathcal{L}}[\Omega, M]$ consists of those functions $\phi: \mathbb{C}^{3} \times U \times \bar{U} \longrightarrow \mathbb{C}$ such that

$$
\phi\left(1+M e^{i \theta}, \frac{k M}{M+e^{-i \theta}}, \frac{k M+L e^{-i \theta}}{M+e^{-i \theta}}-\left(\frac{k M}{M+e^{-i \theta}}\right)^{2} ; z, \zeta\right) \notin \Omega,
$$

whenever $z \in U, \zeta \in \bar{U}, \theta \in \mathbb{R}, \operatorname{Re}\left\{L e^{-i \theta}\right\} \geq k(k-1) M$ for all $\theta$ and $k \geq 1$.

Corollary 2.3. Let $\phi \in \Phi_{\mathcal{L}}[\Omega, M]$. If $f \in \mathcal{A}$ satisfies

$$
\begin{aligned}
& \phi\left(\frac{2 z\left(f^{\prime}(z)\right)^{\lambda}}{f(z)-f(-z)}, 1+\frac{\lambda z f^{\prime \prime}(z)}{f^{\prime}(z)}-\frac{z(f(z)-f(-z))^{\prime}}{f(z)-f(-z)}, \frac{\lambda z^{2} f^{\prime \prime \prime}(z)}{f^{\prime}(z)}\right. \\
& +\frac{\lambda z f^{\prime \prime}(z)}{f^{\prime}(z)}\left(1-\frac{z f^{\prime \prime}(z)}{f^{\prime}(z)}\right)-\frac{z^{2}(f(z)-f(-z))^{\prime \prime}}{f(z)-f(-z)}+\frac{z(f(z)-f(-z))^{\prime}}{f(z)-f(-z)} \times \\
& \left.\times\left(\frac{z(f(z)-f(-z))^{\prime}}{f(z)-f(-z)}-1\right) ; z, \zeta\right) \in \Omega,
\end{aligned}
$$

then

$$
\left|\frac{2 z\left(f^{\prime}(z)\right)^{\lambda}}{f(z)-f(-z)}-1\right|<M
$$

When $\Omega=q(U)=\{w:|w-1|<M\}$, the class $\Phi_{\mathcal{L}}[\Omega, M]$ is simply denoted by $\Phi_{\mathcal{L}}[M]$, then corollary 2.3 takes the following form:

Corollary 2.4. Let $\phi \in \Phi_{\mathcal{L}}[M]$. If $f \in \mathcal{A}$ satisfies

$$
\begin{aligned}
& \mid \phi\left(\frac{2 z\left(f^{\prime}(z)\right)^{\lambda}}{f(z)-f(-z)}, 1+\frac{\lambda z f^{\prime \prime}(z)}{f^{\prime}(z)}-\frac{z(f(z)-f(-z))^{\prime}}{f(z)-f(-z)}, \frac{\lambda z^{2} f^{\prime \prime \prime}(z)}{f^{\prime}(z)}\right. \\
& +\frac{\lambda z f^{\prime \prime}(z)}{f^{\prime}(z)}\left(1-\frac{z f^{\prime \prime}(z)}{f^{\prime}(z)}\right)-\frac{z^{2}(f(z)-f(-z))^{\prime \prime}}{f(z)-f(-z)}+\frac{z(f(z)-f(-z))^{\prime}}{f(z)-f(-z)} \times \\
& \left.\times\left(\frac{z(f(z)-f(-z))^{\prime}}{f(z)-f(-z)}-1\right) ; z, \zeta\right)-1 \mid<M,
\end{aligned}
$$

then

$$
\left|\frac{2 z\left(f^{\prime}(z)\right)^{\lambda}}{f(z)-f(-z)}-1\right|<M
$$

Example 2.1. If $M>0$ and $f \in \mathcal{A}$ satisfies

$$
\begin{aligned}
& \mid \frac{\lambda z^{2} f^{\prime \prime \prime}(z)}{f^{\prime}(z)}-\lambda\left(\frac{z f^{\prime \prime}(z)}{f^{\prime}(z)}\right)^{2}-\frac{z^{2}(f(z)-f(-z))^{\prime \prime}}{f(z)-f(-z)}+ \\
&+\left(\frac{z(f(z)-f(-z))^{\prime}}{f(z)-f(-z)}\right)^{2} \mid<M
\end{aligned}
$$


then

$$
\left|\frac{2 z\left(f^{\prime}(z)\right)^{\lambda}}{f(z)-f(-z)}-1\right|<M .
$$

This implication follows from Corollary 2.4 by taking $\phi(u, v, w ; z, \zeta)=w-$ $v+2$.

Example 2.2. If $M>0$ and $f \in \mathcal{A}$ satisfies

$$
\left|\frac{\lambda z f^{\prime \prime}(z)}{f^{\prime}(z)}-\frac{z(f(z)-f(-z))^{\prime}}{f(z)-f(-z)}\right|<\frac{M}{M+1}
$$

then

$$
\left|\frac{2 z\left(f^{\prime}(z)\right)^{\lambda}}{f(z)-f(-z)}-1\right|<M .
$$

This implication follows from Corollary 2.3 by taking $\phi(u, v, w ; z, \zeta)=v$ and $\Omega=h(U)$ where $h(z)=\frac{M}{M+1} z, M>0$. To apply Corollary 2.3 , we need to show that $\phi \in \Phi_{\mathcal{L}}[\Omega, M]$, that is the admissibility condition (11) is satisfied follows from

$$
\begin{gathered}
\left|\phi\left(1+M e^{i \theta}, \frac{k M}{M+e^{-i \theta}}, \frac{k M+L e^{-i \theta}}{M+e^{-i \theta}}-\left(\frac{k M}{M+e^{-i \theta}}\right)^{2} ; z, \zeta\right)\right|= \\
=\frac{k M}{M+1} \geq \frac{M}{M+1},
\end{gathered}
$$

for $z \in U, \zeta \in \bar{U}, \theta \in \mathbb{R}$ and $k \geq 1$.

\section{Strong Superordination Results}

In this section, we establish strong differential superordination. For this purpose the class of admissible functions given in the following definition will be required.

Definition 3.1. Let $\Omega$ be a set in $\mathbb{C}$ and $q \in \mathcal{H}$. The class of admissible functions $\Phi_{\mathcal{L}}^{\prime}[\Omega, q]$ consists of those functions $\phi: \mathbb{C}^{3} \times U \times \bar{U} \longrightarrow \mathbb{C}$ that satisfy the admissibility condition: $\phi(u, v, w ; \xi, \zeta) \in \Omega$, whenever $u=q(z), \quad v=\frac{z q^{\prime}(z)}{m q(z)}, q(z) \neq 0 \quad$ and $\quad \operatorname{Re}\left\{\frac{w+v^{2}}{v}\right\} \leq \frac{1}{m} \operatorname{Re}\left\{\frac{z q^{\prime \prime}(z)}{q^{\prime}(z)}+1\right\}$, where $z \in U, \zeta \in \bar{U}, \xi \in \partial U$ and $m \geq 1$.

Theorem 3.1. Let $\phi \in \Phi_{\mathcal{L}}^{\prime}[\Omega, q]$. If $f \in \mathcal{A}, \frac{2 z\left(f^{\prime}(z)\right)^{\lambda}}{f(z)-f(-z)} \in Q_{1}$ and

$$
\begin{aligned}
& \phi\left(\frac{2 z\left(f^{\prime}(z)\right)^{\lambda}}{f(z)-f(-z)}, 1+\frac{\lambda z f^{\prime \prime}(z)}{f^{\prime}(z)}-\frac{z(f(z)-f(-z))^{\prime}}{f(z)-f(-z)}, \frac{\lambda z^{2} f^{\prime \prime \prime}(z)}{f^{\prime}(z)}\right. \\
& +\frac{\lambda z f^{\prime \prime}(z)}{f^{\prime}(z)}\left(1-\frac{z f^{\prime \prime}(z)}{f^{\prime}(z)}\right)-\frac{z^{2}(f(z)-f(-z))^{\prime \prime}}{f(z)-f(-z)}+\frac{z(f(z)-f(-z))^{\prime}}{f(z)-f(-z)} \times
\end{aligned}
$$




$$
\left.\times\left(\frac{z(f(z)-f(-z))^{\prime}}{f(z)-f(-z)}-1\right) ; z, \zeta\right)
$$

is univalent in $U$, then

$$
\begin{aligned}
& \Omega \subset\left\{\phi \left(\frac{2 z\left(f^{\prime}(z)\right)^{\lambda}}{f(z)-f(-z)}, 1+\frac{\lambda z f^{\prime \prime}(z)}{f^{\prime}(z)}-\frac{z(f(z)-f(-z))^{\prime}}{f(z)-f(-z)}, \frac{\lambda z^{2} f^{\prime \prime \prime}(z)}{f^{\prime}(z)}\right.\right. \\
& +\frac{\lambda z f^{\prime \prime}(z)}{f^{\prime}(z)}\left(1-\frac{z f^{\prime \prime}(z)}{f^{\prime}(z)}\right)-\frac{z^{2}(f(z)-f(-z))^{\prime \prime}}{f(z)-f(-z)}+\frac{z(f(z)-f(-z))^{\prime}}{f(z)-f(-z)} \times \\
& \left.\left.\times\left(\frac{z(f(z)-f(-z))^{\prime}}{f(z)-f(-z)}-1\right) ; z, \zeta\right): z \in U, \zeta \in \bar{U}\right\},
\end{aligned}
$$

implies

$$
q(z) \prec \frac{2 z\left(f^{\prime}(z)\right)^{\lambda}}{f(z)-f(-z)} .
$$

Proof. Let $p$ defined by (4) and $\psi\left(p(z), z p^{\prime}(z), z^{2} p^{\prime \prime}(z) ; z, \zeta\right)$ defined by (8). Since $\phi \in \Phi_{\mathcal{L}}^{\prime}[\Omega, q]$, from (8) and (12), we have

$$
\Omega \subset\left\{\psi\left(p(z), z p^{\prime}(z), z^{2} p^{\prime \prime}(z) ; z, \zeta\right): z \in U, \zeta \in \bar{U}\right\} .
$$

From (7), we see that the admissibility condition for $\phi \in \Phi_{\mathcal{L}}^{\prime}[\Omega, q]$ is equivalent to the admissibility condition for $\psi$ as given in Definition 1.5. Hence $\psi \in \Psi^{\prime}[\Omega, q]$ and by Lemma 1.2, $q(z) \prec p(z)$ or equivalently

$$
q(z) \prec \frac{2 z\left(f^{\prime}(z)\right)^{\lambda}}{f(z)-f(-z)} .
$$

We consider the special situation when $\Omega \neq \mathbb{C}$ is a simply connected domain. In this case $\Omega=h(U)$, for some conformal mapping $h$ of $U$ onto $\Omega$ and the class $\Phi_{\mathcal{L}}^{\prime}[h(U), q]$ is written as $\Phi_{\mathcal{L}}^{\prime}[h, q]$. The following result is an immediate consequence of Theorem 3.1.

Theorem 3.2. Let $\phi \in \Phi_{\mathcal{L}}^{\prime}[h, q], q \in \mathcal{H}$ and $h$ be analytic in $U$. If $f \in \mathcal{A}$, $\frac{2 z\left(f^{\prime}(z)\right)^{\lambda}}{f(z)-f(-z)} \in Q_{1}$ and

$$
\begin{aligned}
& \phi\left(\frac{2 z\left(f^{\prime}(z)\right)^{\lambda}}{f(z)-f(-z)}, 1+\frac{\lambda z f^{\prime \prime}(z)}{f^{\prime}(z)}-\frac{z(f(z)-f(-z))^{\prime}}{f(z)-f(-z)}, \frac{\lambda z^{2} f^{\prime \prime \prime}(z)}{f^{\prime}(z)}\right. \\
& +\frac{\lambda z f^{\prime \prime}(z)}{f^{\prime}(z)}\left(1-\frac{z f^{\prime \prime}(z)}{f^{\prime}(z)}\right)-\frac{z^{2}(f(z)-f(-z))^{\prime \prime}}{f(z)-f(-z)}+\frac{z(f(z)-f(-z))^{\prime}}{f(z)-f(-z)} \times \\
& \left.\times\left(\frac{z(f(z)-f(-z))^{\prime}}{f(z)-f(-z)}-1\right) ; z, \zeta\right)
\end{aligned}
$$


is univalent in $U$, then

$$
\begin{aligned}
& h(z) \prec \prec \phi\left(\frac{2 z\left(f^{\prime}(z)\right)^{\lambda}}{f(z)-f(-z)}, 1+\frac{\lambda z f^{\prime \prime}(z)}{f^{\prime}(z)}-\frac{z(f(z)-f(-z))^{\prime}}{f(z)-f(-z)}, \frac{\lambda z^{2} f^{\prime \prime \prime}(z)}{f^{\prime}(z)}\right. \\
& +\frac{\lambda z f^{\prime \prime}(z)}{f^{\prime}(z)}\left(1-\frac{z f^{\prime \prime}(z)}{f^{\prime}(z)}\right)-\frac{z^{2}(f(z)-f(-z))^{\prime \prime}}{f(z)-f(-z)}+\frac{z(f(z)-f(-z))^{\prime}}{f(z)-f(-z)} \times \\
& \left.\times\left(\frac{z(f(z)-f(-z))^{\prime}}{f(z)-f(-z)}-1\right) ; z, \zeta\right)
\end{aligned}
$$

implies

$$
q(z) \prec \frac{2 z\left(f^{\prime}(z)\right)^{\lambda}}{f(z)-f(-z)} .
$$

By taking $\phi(u, v, w ; z, \zeta)=u+\frac{v}{\beta u+\gamma},(\beta, \gamma \in \mathbb{C})$ in Theorem 3.2, we state the following corollary:

Corollary 3.1. Let $\beta, \gamma \in \mathbb{C}$ and let $h$ be convex in $U$ with $h(0)=1$. Suppose that the differential equation $q(z)+\frac{z q^{\prime}(z)}{\beta q(z)+\gamma}=h(z)$ has a univalent solution $q$ that satisfies $q(0)=1$ and $q(z) \prec h(z)$. If $f \in \mathcal{A}, \frac{2 z\left(f^{\prime}(z)\right)^{\lambda}}{f(z)-f(-z)} \in$ $\mathcal{H} \cap Q_{1}$ and

$$
\frac{2 z\left(f^{\prime}(z)\right)^{\lambda}}{f(z)-f(-z)}+\frac{\left(1+\frac{\lambda z f^{\prime \prime}(z)}{f^{\prime}(z)}\right)(f(z)-f(-z))-z(f(z)-f(-z))^{\prime}}{2 \beta z\left(f^{\prime}(z)\right)^{\lambda}+\gamma(f(z)-f(-z))}
$$

is univalent in $U$, then

$h(z) \prec \prec \frac{2 z\left(f^{\prime}(z)\right)^{\lambda}}{f(z)-f(-z)}+\frac{\left(1+\frac{\lambda z f^{\prime \prime}(z)}{f^{\prime}(z)}\right)(f(z)-f(-z))-z(f(z)-f(-z))^{\prime}}{2 \beta z\left(f^{\prime}(z)\right)^{\lambda}+\gamma(f(z)-f(-z))}$,

implies

$$
q(z) \prec \frac{2 z\left(f^{\prime}(z)\right)^{\lambda}}{f(z)-f(-z)} .
$$

The next result gives the best subordinant of the strong differential superordination (13):

Theorem 3.3. Let $h$ be analytic in $U$ and $\phi: \mathbb{C}^{3} \times U \times \bar{U} \longrightarrow \mathbb{C}$. Suppose that the differential equation

$$
\phi\left(q(z), \frac{z q^{\prime}(z)}{q(z)}, \frac{z^{2} q^{\prime \prime}(z)}{q(z)}+\frac{z q^{\prime}(z)}{q(z)}-\left(\frac{z q^{\prime}(z)}{q(z)}\right)^{2} ; z, \zeta\right)=h(z)
$$

has a solution $q \in Q_{1}$. If $\phi \in \Phi_{\mathcal{L}}^{\prime}[h, q], f \in \mathcal{A}, \frac{2 z\left(f^{\prime}(z)\right)^{\lambda}}{f(z)-f(-z)} \in Q_{1}$ and

$$
\phi\left(\frac{2 z\left(f^{\prime}(z)\right)^{\lambda}}{f(z)-f(-z)}, 1+\frac{\lambda z f^{\prime \prime}(z)}{f^{\prime}(z)}-\frac{z(f(z)-f(-z))^{\prime}}{f(z)-f(-z)}, \frac{\lambda z^{2} f^{\prime \prime \prime}(z)}{f^{\prime}(z)}\right.
$$




$$
\begin{aligned}
& +\frac{\lambda z f^{\prime \prime}(z)}{f^{\prime}(z)}\left(1-\frac{z f^{\prime \prime}(z)}{f^{\prime}(z)}\right)-\frac{z^{2}(f(z)-f(-z))^{\prime \prime}}{f(z)-f(-z)}+\frac{z(f(z)-f(-z))^{\prime}}{f(z)-f(-z)} \times \\
& \left.\times\left(\frac{z(f(z)-f(-z))^{\prime}}{f(z)-f(-z)}-1\right) ; z, \zeta\right)
\end{aligned}
$$

is univalent in $U$, then

$$
\begin{aligned}
& h(z) \prec \prec \phi\left(\frac{2 z\left(f^{\prime}(z)\right)^{\lambda}}{f(z)-f(-z)}, 1+\frac{\lambda z f^{\prime \prime}(z)}{f^{\prime}(z)}-\frac{z(f(z)-f(-z))^{\prime}}{f(z)-f(-z)}, \frac{\lambda z^{2} f^{\prime \prime \prime}(z)}{f^{\prime}(z)}\right. \\
& +\frac{\lambda z f^{\prime \prime}(z)}{f^{\prime}(z)}\left(1-\frac{z f^{\prime \prime}(z)}{f^{\prime}(z)}\right)-\frac{z^{2}(f(z)-f(-z))^{\prime \prime}}{f(z)-f(-z)}+\frac{z(f(z)-f(-z))^{\prime}}{f(z)-f(-z)} \times \\
& \left.\times\left(\frac{z(f(z)-f(-z))^{\prime}}{f(z)-f(-z)}-1\right) ; z, \zeta\right),
\end{aligned}
$$

implies

$$
q(z) \prec \frac{2 z\left(f^{\prime}(z)\right)^{\lambda}}{f(z)-f(-z)},
$$

and $q$ is the best subordinant.

Proof. The proof is similar to that of Theorem 2.4 and is omitted.

\section{SAndwich Results}

By combining Theorem 2.2 and Theorem 3.2, we obtain the following sandwich theorem:

Theorem 4.1. Let $h_{1}$ and $q_{1}$ be analytic functions in $U, h_{2}$ be univalent in $U, q_{2} \in Q_{1}$ with $q_{1}(0)=q_{2}(0)=1$ and $\phi \in \Phi_{\mathcal{L}}\left[h_{2}, q_{2}\right] \cap \Phi_{\mathcal{L}}^{\prime}\left[h_{1}, q_{1}\right]$. If $f \in \mathcal{L}, \frac{2 z\left(f^{\prime}(z)\right)^{\lambda}}{f(z)-f(-z)} \in \mathcal{H} \cap Q_{1}$ and

$$
\begin{aligned}
& \phi\left(\frac{2 z\left(f^{\prime}(z)\right)^{\lambda}}{f(z)-f(-z)}, 1+\frac{\lambda z f^{\prime \prime}(z)}{f^{\prime}(z)}-\frac{z(f(z)-f(-z))^{\prime}}{f(z)-f(-z)}, \frac{\lambda z^{2} f^{\prime \prime \prime}(z)}{f^{\prime}(z)}\right. \\
& +\frac{\lambda z f^{\prime \prime}(z)}{f^{\prime}(z)}\left(1-\frac{z f^{\prime \prime}(z)}{f^{\prime}(z)}\right)-\frac{z^{2}(f(z)-f(-z))^{\prime \prime}}{f(z)-f(-z)}+\frac{z(f(z)-f(-z))^{\prime}}{f(z)-f(-z)} \times \\
& \left.\times\left(\frac{z(f(z)-f(-z))^{\prime}}{f(z)-f(-z)}-1\right) ; z, \zeta\right)
\end{aligned}
$$

is univalent in $U$, then

$$
\begin{aligned}
& h_{1}(z) \prec \prec \phi\left(\frac{2 z\left(f^{\prime}(z)\right)^{\lambda}}{f(z)-f(-z)}, 1+\frac{\lambda z f^{\prime \prime}(z)}{f^{\prime}(z)}-\frac{z(f(z)-f(-z))^{\prime}}{f(z)-f(-z)}, \frac{\lambda z^{2} f^{\prime \prime \prime}(z)}{f^{\prime}(z)}\right. \\
& +\frac{\lambda z f^{\prime \prime}(z)}{f^{\prime}(z)}\left(1-\frac{z f^{\prime \prime}(z)}{f^{\prime}(z)}\right)-\frac{z^{2}(f(z)-f(-z))^{\prime \prime}}{f(z)-f(-z)}+\frac{z(f(z)-f(-z))^{\prime}}{f(z)-f(-z)} \times
\end{aligned}
$$




$$
\left.\times\left(\frac{z(f(z)-f(-z))^{\prime}}{f(z)-f(-z)}-1\right) ; z, \zeta\right) \prec \prec h_{2}(z),
$$

implies

$$
q_{1}(z) \prec \frac{2 z\left(f^{\prime}(z)\right)^{\lambda}}{f(z)-f(-z)} \prec q_{2}(z) .
$$

By combining Corollary 2.1 and Corollary 3.1, we obtain the following sandwich corollary:

Corollary 4.1. Let $\beta, \gamma \in \mathbb{C}$ and let $h_{1}, h_{2}$ be convex in $U$ with $h_{1}(0)=$ $h_{2}(0)=1$. Suppose that the differential equations

$$
q_{1}(z)+\frac{z q_{1}^{\prime}(z)}{\beta q_{1}(z)+\gamma}=h_{1}(z), \quad q_{2}(z)+\frac{z q_{2}^{\prime}(z)}{\beta q_{2}(z)+\gamma}=h_{2}(z)
$$

have a univalent solutions $q_{1}$ and $q_{2}$, respectively, that satisfy

$$
q_{1}(0)=q_{2}(0)=1 \quad \text { and } \quad q_{1}(z) \prec h_{1}(z), q_{2}(z) \prec h_{2}(z) .
$$

If $f \in \mathcal{A}, \frac{2 z\left(f^{\prime}(z)\right)^{\lambda}}{f(z)-f(-z)} \in \mathcal{H} \cap Q_{1}$ and

$$
\frac{2 z\left(f^{\prime}(z)\right)^{\lambda}}{f(z)-f(-z)}+\frac{\left(1+\frac{\lambda z f^{\prime \prime}(z)}{f^{\prime}(z)}\right)(f(z)-f(-z))-z(f(z)-f(-z))^{\prime}}{2 \beta z\left(f^{\prime}(z)\right)^{\lambda}+\gamma(f(z)-f(-z))}
$$

is univalent in $U$, then

$$
\begin{aligned}
h_{1}(z) & \prec \prec \frac{2 z\left(f^{\prime}(z)\right)^{\lambda}}{f(z)-f(-z)}+\frac{\left(1+\frac{\lambda z f^{\prime \prime}(z)}{f^{\prime}(z)}\right)(f(z)-f(-z))-z(f(z)-f(-z))^{\prime}}{2 \beta z\left(f^{\prime}(z)\right)^{\lambda}+\gamma(f(z)-f(-z))} \\
& \prec \prec h_{2}(z)
\end{aligned}
$$

implies

$$
q_{1}(z) \prec \frac{2 z\left(f^{\prime}(z)\right)^{\lambda}}{f(z)-f(-z)} \prec q_{2}(z)
$$

\section{REFERENCES}

[1] M. K. Aouf, R. M. El-Ashwah, A. M. Abd-Eltawab, Differential subordination and superordination results of higher-order derivatives of p-valent functions involving a generalized differential operator, Southeast Asian Bulletin of Mathematics, 36 (4) (2012), 475-488.

[2] K. O. Babalola, On $\lambda$-pseudo-starlike functions, Journal of Classical Analysis, 3 (2) (2013), 137-147.

[3] N. E. Cho, O. S. Kwon, H. M. Srivastava, Strong differential subordination and superordination for multivalently meromorphic functions involving the Liu-Srivastava operator, Integral Transforms and Special Functions, 21 (8) (2010), 589-601.

[4] M. P. Jeyaraman, T. K. Suresh, Strong differential subordination and superordination of analytic functions, Journal of Mathematical Analysis and Applications, 385 (2) (2012), 854-864. 
[5] S. S. Miller, P. T. Mocanu, Second order differential inequalities in the complex plane, Journal of Mathematical Analysis and Applications, 65 (1978), 289-305.

[6] S. S. Miller, P. T. Mocanu, Differential Subordinations: Theory and Applications, Series on Monographs and Textbooks in Pure and Applied Mathematics, Vol. 225, Marcel Dekker, New York, 2000.

[7] S. S. Miller, P. T. Mocanu, Subordinants of differential superordinations, Complex Variables, Theory and Application, 48 (10) (2003), 815-826.

[8] G. I. Oros, Strong differential superordination, Acta Universitatis Apulensis, 19 (2009), 101-106.

[9] G. I. Oros, Gh. Oros, Strong differential subordination, Turkish Journal of Mathematics, 33 (3) (2009), 249-257.

[10] K. Sakaguchi, On a certain univalent mapping, Journal of the Mathematical Society of Japan, 11 (1) (1959), 72-75.

[11] A. K. Wanas, A. L. Alina, On a new strong differential subordinations and superordinations of analytic functions involving the generalized operator, International Journal of Pure and Applied Mathematics, 116 (3) (2017), 571-579.

[12] A. K. Wanas, A. H. Majeed, New strong differential subordinations and superordinations of symmetric analytic functions, International Journal of Mathematical Analysis, 11 (11) (2017), 543-549.

[13] A. K. Wanas, A. H. Majeed, Strong differential subordinations for higher-order derivatives of multivalent analytic functions defined by linear operator, Khayyam Journal of Mathematics, 3 (2) (2017), 160-171.

H. M. Srivastava

Department of Mathematics and Statistics

UNIVERSITY OF VICTORIA

Victoria, British Columbia V8W 3R4

CANAdA

Department of Medical Research

China Medical University Hospital

China Medical University

TAICHUNG 40402

Taiwan, Republic of China

E-mail address: harimsri@math.uvic.ca

A. K. WANAS

Department of Mathematics

College of Science

University of Al-QAdisiyah

IRAQ

E-mail address: abbas.kareem.w@qu.edu.iq 